\title{
THE GATTINI CAMERAS FOR OPTICAL SKY BRIGHTNESS MEASUREMENTS AT DOME C, ANTARCTICA
}

\author{
A. Moore ${ }^{1}$, E. Aristidi ${ }^{2}$, M. Ashley ${ }^{3}$, R. Briguglio ${ }^{4}$, M. Busso $^{5}$, \\ M. Candidi ${ }^{6}$, J. Everett ${ }^{3}$, S. Kenyon ${ }^{3}$, J. Lawrence ${ }^{3}$, B. Le Roux ${ }^{7}$, \\ D. Luong-Van ${ }^{3}$, A. Phillips ${ }^{3}$, R. Ragazzoni ${ }^{8}$, P. Salinari ${ }^{9}$, J. Storey ${ }^{3}$, \\ M. Taylor ${ }^{3}$, G. Tosti ${ }^{10}$ and T. Travouillon ${ }^{1}$
}

\begin{abstract}
The Gattini cameras are two site testing instruments for the measurement of optical sky brightness, large area cloud cover and auroral detection of the night sky above the high altitude Dome $\mathrm{C}$ site in Antarctica. The cameras have been operating since installation in January 2006 and are currently at the end of the first Antarctic winter season. The cameras are transit in nature and are virtually identical, both adopting Apogee Alta CCD detectors. By taking frequent images of the night sky we obtain long term cloud cover statistics, measure the sky background intensity as a function of solar and lunar altitude and phase and directly measure the spatial extent of bright aurora if present and when they occur. The full data set will return in December 2006 however a limited amount of data has been transferred via the Iridium network enabling preliminary data reduction and system evaluation. An update of the project is presented together with preliminary results from data taken since commencement of the winter season.
\end{abstract}

\footnotetext{
${ }^{1}$ California Institute of Technology, Pasadena, CA 91106, USA e-mail: amoore@astro.caltech.edu

${ }^{2}$ LUAN, Université de Nice, Parc Valrose, 06108 Nice Cedex 2, France

3 School of Physics, University of New South Wales, Sydney, NSW 2052, Australia

${ }^{4}$ Dipartimento di Fisica, Univerita La Sapienza. Piazzale Aldo Moro, 00185 Rome, Italy

5 Dipartimento di Fisica, Universita di Perugia, via A. Pascoli, 06123 Perugia, Italy

${ }^{6}$ Istituto di Fisica dello Spazio, CNR, via Fosso Cavaliere 100, Roma, and Programma Nazionale di Ricerche in Antartide, ENEA, Roma, Italy

7 Observatoire Astronomique de Marseille, 2 place Leverrier, 1.3248 Marseille, France

* INAF: Osservatorio Astronomico di Padova, Vicolo dell'Osservatorio 5, 35122 Padova, Italy

9 INAF: Osservatorio Astrofisico di Arcetri, Largo Enrico Fermi 5, 50125 Firenze, Italy

10 Dipartimento di Fisica. Universita di Perugia, via A. Pascoli, 06123 Perugia, Italy
} 


\section{Introduction}

Since the release of spectacular seeing measurements at visible wavelengths $(550 \mathrm{~nm})$ taken at the Antarctic site of Dome $\mathrm{C}$ during summertime at ground level (Aristidi et al. 2005) and during winter-time (Lawrence et al. 2004) there have been several proposals for $2 \mathrm{~m}$ class optical telescopes that wish to exploit this mique location (such as Ragazzoni et al. 2005; Lawrence et al. 2005; Lloyd et al. 2004). Optical sky background measurements, that include twilight and aurora, have largely been unquantified for this site. Given the latitude of Dome C $\left(-75^{\circ}\right)$ the site experiences short periods of twilight diumally throughout the winter season, unlike the South Pole that undergoes an extended period of true astronomical dark time (defined by a solar altitude less than $-18^{\circ}$ ). An estimate of the true amount of dark time experienced above this site is important for any proposing optical facility. An excellent summary of the optical sky brightness modelled by constituent part for the Dome C site can be found in Kenyon et al. (2006).

The cameras provide a method to: (1) calculate optical sky brightness as a function of lunar phase, altitude and distance to target; (2) calculate cloud cover across a large area of sky; $(3)$ detect spatially bright auroral events if and when they occur and; (4) estimate the optical extinction.

\section{Gattini-SBC}

Gattini-SBC, shown in Figure 1 (left), images a $6^{\circ}$ by $4^{\circ}$ field of view centred close to the South Pole (zenith angle of $15^{\circ}$ at the Dome $\mathrm{C}$ site). The primary goal is to measure accurately the contribution to the optical sky background by integrating sky photons in between the resolved stars on the CCD.

In summary the Gattini-SBC (1) images a field $6^{\circ}$ by $4^{\circ}$ centered on the SP every $5 \mathrm{~min}$; (2) alternates between $8 \mathrm{~s}$ and $40 \mathrm{~s}$ exp time every 5 min avoiding star trails (though not a mandatory requirement); (3) identifies bright stars and uses for ftux calibration of sky pixel intensity; (4) produces a faint 2D sky brightness map every $10 \mathrm{~min}$ from the $40 \mathrm{~s}$ data set and; (5) as a side project-variable star monitoring and searching for planetary transits from $8 \mathrm{~s}$ data set.

The primary aim of Gattini-SBC is the accurate measmement of sky background magnitude at optical wavelengths above the Dome $\mathrm{C}$ site. To be able to integrate sky background photons without stellar contamination the plate scale must be small enough such that the stellar density on the CCD is small but not small enough that the individual stars cannot be identified and removed. Identification is essential as it provides a direct calibration source for the sky background photons. To aid greatly in this task the FoV of the camera is centred roughly on the South Pole.

A Nikor $1 / 135 \mathrm{~mm}$ lens was chosen resulting in a plate scale of $\approx 11$ aresec/pix. Set at maximum stop diameter, the aperture of the Gattini-SBC camera is $\approx 50 \mathrm{~mm}$ $(\mathrm{F} / 2.8)$. We have chosen to image through a sloan $g$ fiter that is attached to the front of the lens for meaningful comparison to other sites $(477 \mathrm{~nm}$ central wavelength) and to concentrate on determining solar and lunar contributions to 

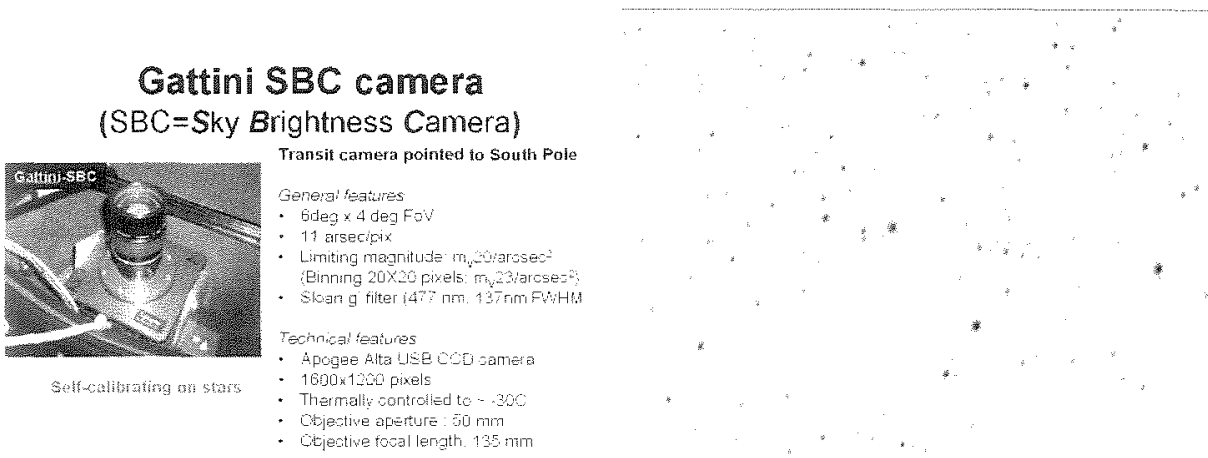

Fig. 1. A summary of the Gattini-SBC camera (Sky Brightness Camera) shown inside the molosure (left). The camera measures the sloan $g^{\prime}(477 \mathrm{~nm})$ sky background intensity every 10 min at the Dome $\mathrm{C}$ site. Accurate calibration across the field of view is performed nsing the stars in the field. The camera objective lens is a Nikor $\mathrm{f} / 135 \mathrm{~mm} \mathrm{~F} / 2.8$ set at maximum stop diameter. (Right) raw (negative) image taken with the Gattini-SBC camcra. The image shows a $6^{\circ}$ by $4^{\circ}$ rectangular field centred on the South Pole. Integration time $=40 \mathrm{~s}$. The faintest magnitude star detected is equivalent to $\approx 21 \mathrm{Vmag} / \mathrm{arcsec}^{2}$. binning of small pixel areas is required to reach fainter sky background measurements. Image taken April 8th 2006 at 23:48 local time.

sky background more dominant at this wavelength. A raw image taken with Gattini-SBC is shown in Figure 1 (right).

\section{Gattini-Allsky}

The second instrument is a wide field camera with a primary goal of measuring accurate, frequent and long term cloud statistics at the Dome C site as well as the recording of bright auroral events if and when they occur. The camera, shown in Figure 2 (left), uses a fish-eye lens that in combination with the camera images a field of view $110^{\circ}$ by $90^{\circ}$ roughly centred on local zenith. The camera functions an identical manner to the Gattini-SBC camera though contans no fitter for the 2006 season. A raw image taken with the camera is shown in Figure 2 (right).

\section{Camera Design}

Fach camera uses an identical Apogee Alta U2020 interline camera that is supplied with an intemal Peltier cooler. The CCD chip is a Kodak KAI2020 with 1600 by 12007.4 micron pixels and corresponding quantum efficiency of $\approx 55 \%$ at $477 \mathrm{~mm}$. The interline feature is very important as the system requires no mechanical shutter so maximizing reliability. The Apogee Alta cameras have proved to be extremely reliable. 


\section{Gattini-Allsky}
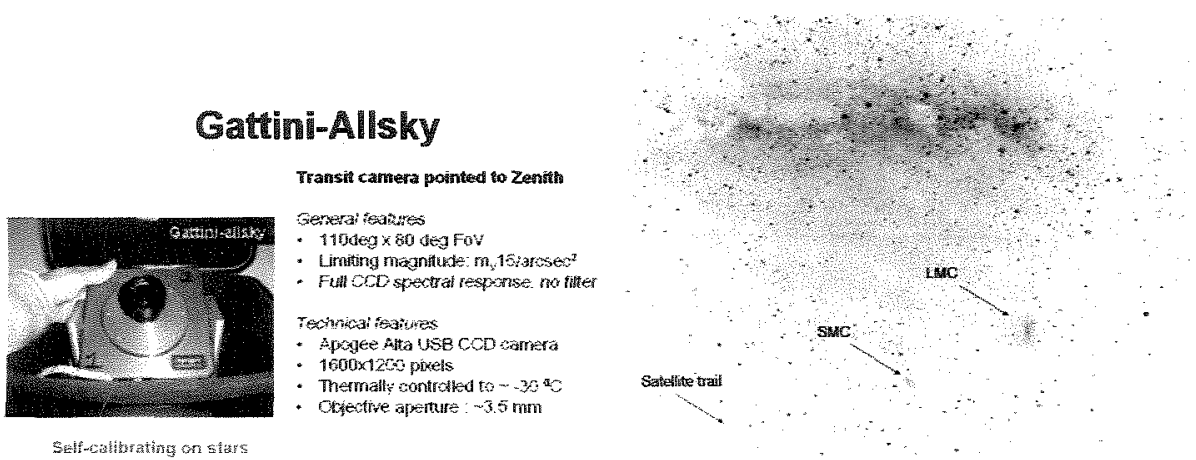

Fig. 2. (Left) a summary of the Gattini-allsky camera (shown left). The cannera acquires broadband images of a large area field centred at local zenith at the Dome $\mathrm{C}$ site. No filter is included in the system. The lens is a Pentax fish-eye $\mathrm{f} / 6 \mathrm{~mm} \mathrm{~F} / 1.4$ with the stop setting at maximum diameter. (Right) a raw (negative) image taken by the Gattini-allsky camera. The image has not been corrected for distortion nor flat field effects (both are significant effects for a fish-eye objective). The $40 \mathrm{~s}$ exposure was taken on March 29 th 2006 during dark time.

A technical description of the Gattini system including thermal and data management, project timcline and romotc data transfer can be found in Moore et al. (2006).

\section{Data Management}

Data storage, transfer and communications are largely handled by the UNSW team in Sydney. A NSF supplied Iridium link enables direct connection to the Gattini supervisor computer. On a day to day basis the process of retrieving system status updates and up to 1MB of data per connection is automated, however, it is possible to change any feature of the system throughout the winter season. This has been extremely useful for changing variables such as camera exposure time and cadence when data is first retrieved. A LAN connection between the Gattini $\mathrm{PC}$ and Concordia station means that an automated back-up occurs, currently set to once per week. The transfer of system status data throughout the season has been a critical element to the success of the Gattini instruments.

\section{Lessons Learnt}

Likely due to substantial pre-testing, both low temperature and site testing, thankfully there are few negatives to report. The components that have worked particularly well are (1) a redundant system: 2 cameras rather than only 1 has been a great feature; (2) data transfer (UNSW/NSF Iridium card); (3) apogee camera 
reliability; (4) automated software (used past experience of UNSW team); (5) heating system and; (6) cabling and connectors.

The most problematic feature by far to report is icing of the Gattini-SBC glass window, in particular when the temperature dropped dramatically in May 2006. Prior to this little icing of the window occurred. Though we are unsure exactly what is the major cause of the icing the following can be said with certainty (1) Pyrex glass has a high emissivity (0.95) therefore is an excellent radiator of heat (2) Pyrex glass does not transmit NIR wavelengths therefore does not pass heat through from the inside to the outside very well, compared to, for example, Calcium Fluoride (3) a flat piece of glass with a large indent round the rim is not ideal for stopping ice and/or snow accumulating (4) a flat piece of glass sees virtually all of the sky that is at a very low temperature and (5) the highly curved acrylic dome from a geometrical point of view has a lower chance of acquiring snow on the outside, or for it to remain very long when it does. It should be noted that the window of the Gattini-allsky camera had virtually no problems with icing nor snow build-up during the season and that in this case the window is a $1 / 8^{\text {th }}$ inch thick acrylic dome. A new window will be incorporated into the GattiniSBC enclosure for the 2007 winter season. This window will include an infra-red transmitting material with no indent and fans for keeping air moving across the inside and outside surfaces of the window.

\section{Preliminary Results}

The complete dataset representing the 2006 winter season will be retrieved from the Dome $\mathrm{C}$ site in December 2006. This includes a FITS format image taken every $10 \mathrm{~min}$ at an exposure time of $8 \mathrm{~s}$ and $40 \mathrm{~s}$ from each camera. The datasets (one for $8 \mathrm{~s}$ and the other for $40 \mathrm{~s}$ ) are interleaved every $5 \mathrm{~min}$. At the time of writing the data accessible is that downloaded remotely via the Iridium satellite network, corresponding to 2 FITS images taken every day. Preliminary results from this data are presented here.

\subsection{Sky Background Variation versus Solar and Lunar Altitude}

Variation of the sky background versus solar altitude is shown in Figure 3 (left) for Gattini-SBC. The $y$-axis units are median pixel flux value as measured by the CCD (accurate conversion to AB magnitude awaits further analysis). For the data plotted distinction is made between observations when the moon is either above or below the horizon. Data reduction on the full data set is required to determine the solar altitude that equates to astronomical dark time at the Dome C site (usually stated as $-18^{\circ}$ ) though found to be slightly less at high altitude sites such as Paranal (Patat el al. 2006). The maximum increase in sky background due to the moon is approximately 50-80 times that of dark sky value.

Equivalent plots for Gattini-allsky are shown in Figure 8. Not intentionally by design the cameras have similar flux gathering power per pixel, as the focal length difference is compensated by the difference in pixel area on the sky. Therefore 

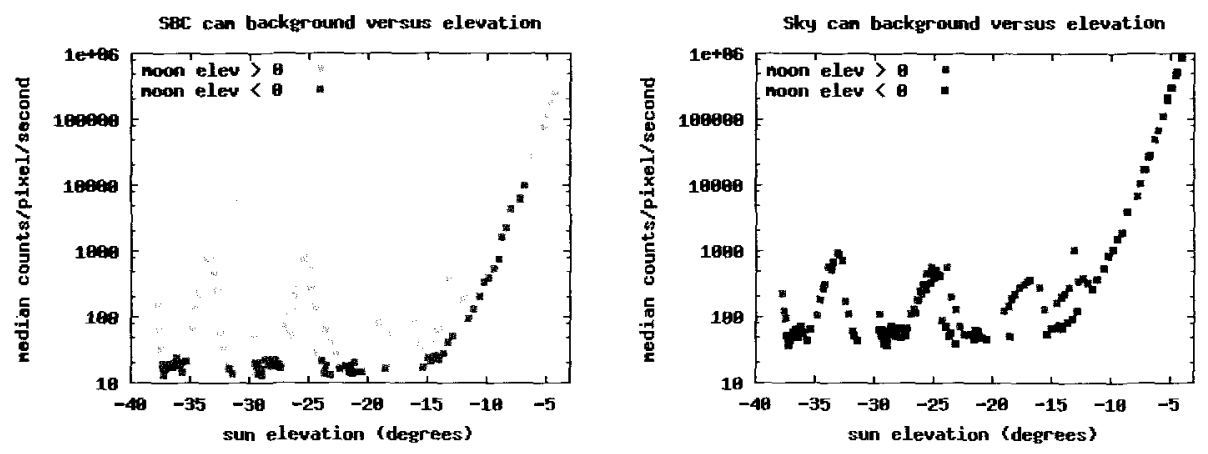

Fig. 3. (Left) Gattini-SBC raw pixel median count rate versus solar elevation. Distinetion is made between measurements taken with a lunar altitude above and below zero. The data shown is calculated from all available images (on average 2 per day since commencement of operation) via the Iridium satellite transfer. This is a factor of 36 times smaller than the number of images stored locally at the site. A thorough data analysis is in progress. (Right) the same plot is shown for Gattini-allsky. Sec text for details.

similar maximum values of intensity of the sky background are seen. As expected. due mostly to star contamination, the minimum flux per pixel moasured is much higher than that of Gattini-SBC.

\subsection{Cloud Cover}

Cloud cover estimates are obtained from the images taken by Gattini-allsky. From preliminary analysis the percentage of cloud-frce nights appears to bo equal or better to the $70 \%$ figure measured by ICECAM (Ashley et al. 2003).

\subsection{Auroral Detection}

The Gattini-allsky camera can directly detect bright auroral events equivalent in magnitude to the limiting magnitude of the Gattini-allsky camera (approximately $M_{V}=16$ ). The ficld of view is approximately $110^{\circ}$ by $80^{\circ}$ therefore aurora occurring outside this field, for cxample towards the active ring located towards the horizon at the Dome $\mathrm{C}$ site, are not detected. So far only 1 such event has been detected from the preliminary dataset so far, however it should be noted that this dataset represents a very small fraction of temporal coverage and more accurate statistics will be derived from the full dataset.

\section{Upgrades for Winter 2007}

For the upconing winter 2007 season the following changes will be made to the Gattini instruments: (1) the instruments will be relocated to the BRAIN shed at Dome C though remaining a reasonable distance from the possibly light polluting 
Concordia station; (2) the window of the Gattini-SBC will be changed for a version that hopefully will prevent ice build-up on the inside and outside surfaces; (3) a baffle will be installed on the Gattini-allsky camera to prevent lunar contamination by reducing the field of view to roughly 900 and; (4) minor hardware changes such as increased hard disk space from $200 \mathrm{~GB}$ to $500 \mathrm{~GB}$ will be performed.

The Gattini team would jike to thank greatly the following institutions for theis continued support of the Gattini projects: (1) PNRA (Programma Narionale di Ricorche in Antartide): (2) TPEV (Institut Polaive Français Paul Emile Victor) and PNRA staff assistance at tho Concordia station:

(3) the National Science Fomdation for supply of an Iridium card and logistical assistance and:

(4) the Australian Alltarctic Division.

\section{References}

Aristidi. E., ct al., 2005. A\&A, 414, 651A

Lawrence, J.S.. Ashley, M.C.B., Tokovinin, A.. \& Travouillon. T.. 2004. Nature, 431. 278.

Ragazzoni. R., ct al., 2005. EAS Publ. Scr., 14, 161

Lawrence, J.S.. Burton, M.G. Ashley, M.C.B., \& Storey, J.W.V.. 2005. FAS Publ. Ser.. 14,321

Lloyd. J.P., Swain, M., van Belle. G.T., et al. 2004. Amer. Astron. Soc. Meet. Abs. 20j, 181.03

Kenyon, S.L.. \& Storey, J.W.V., 2006, PASP, 118, 489

Moore, A.M., et al., 2006, SPIE, 6267

Patat, F., Ugolnikov, O.S., \& Postylyakov, O.V., 2006, A\& A, 455, 385

Ashley, M.C.B., Storey, J.W., \& Burton, M.G., 2003, Astronomy in Antarctica, 25th meeting of the LAU, Special Session 2, 18 .July, 2003 in Sydney. Australia. meeting abstract. 2 